\title{
A Photometric Study of the Contact Binaries: XY Leo, EE Cet and AQ Psc
}

\author{
Gojko Djurašević $^{\mathrm{A}}$, Dinko Dimitrov ${ }^{\mathrm{B}}$, Bojan Arbutina ${ }^{\mathrm{A}}$, Berahitdin Albayrak $^{\mathrm{C}, \mathrm{E}}$, \\ Selim Osman Selam ${ }^{\mathrm{C}}$ and Olga Atanacković-Vukmanović ${ }^{\mathrm{D}}$ \\ A Astronomical Observatory, Volgina 7, 11160 Belgrade, Serbia \\ ${ }^{B}$ Institute of Astronomy, Bulgarian Academy of Sciences, Tzarigradsko Chosse 72, 1784 Sofia, Bulgaria \\ C Ankara University, Faculty of Science, Department of Astronomy and Space Sciences, 06100, Tandoğan, \\ Ankara, Turkey \\ D Department of Astronomy, Faculty of Mathematics, University of Belgrade, Studentski trg 16, 11000 \\ Belgrade, Serbia \\ E Corresponding author. E-mail: albayrak@astro1.science.ankara.edu.tr
}

Received 2006 June 9, accepted 2007 February 19

\begin{abstract}
New photoelectric $B V$ light curves of three close eclipsing binaries XY Leo, EE Cet and AQ Psc were observed and studied with the aim to derive the physical parameters of these systems. The following results were obtained: (a) the W-type contact binary system XY Leo is in a marginal overcontact configuration $\left(f_{\text {over }} \sim 2.4 \%\right.$ ) with a relatively large temperature difference between the components $(\sim 330 \mathrm{~K})$; (b) due to the proximity of the companion of EE Cet in the visual binary ADS 2163, the light curves of EE Cet were contaminated by the third light, having a relatively large impact on the system-parameter estimates, and the solutions made with the third light parameter, $L_{3} \sim 0.54$, describe EE Cet as a high-overcontact $\left(f_{\text {over }} \sim 33 \%\right.$ ) W-type system; (c) the A-type W UMa contact eclipsing binary AQ Psc is in an overcontact configuration $\left(f_{\text {over }} \sim 20 \%\right)$. The relatively small temperature difference $(\sim 150 \mathrm{~K})$ and large difference in component masses suggest a significant energy transfer through the connecting neck of the common envelope. The absolute system parameters are obtained by combining our photometric solution with the spectroscopic elements given by other authors.
\end{abstract}

Keywords: binaries: close — binaries: eclipsing — stars: fundamental parameters — stars: individual: XY Leo, EE Cet, AQ Psc

\section{Introduction}

\subsection{XY Leo}

The eclipsing binary system XY Leo $(P \sim 0.28$ days $)$ was discovered by Hoffmeister (1934). Many photometric studies made in the past show the light-curve asymmetries and variability (see e.g. Hilditch 1981; Kaluzny \& Pojmanski 1983; Hrivnak 1985) attributed to the asymmetric distribution of cool spot regions on one or both components. The orbital period of the system changes with time, and $\mathrm{O}-\mathrm{C}$ residuals, indicating the differences between the observed times of eclipses and the calculated ones using the light elements, were studied by Gehlich, Prolss \& Wehmeyer (1972), Kaluzny \& Pojmanski (1983), Hrivnak (1985), Pan \& Cao (1998) and recently by Yakut et al. (2003). In the latter paper it was shown that the orbital period of the system oscillates with a period of 19.6 years, with a semi-amplitude of 0.023 days. This sine-like regular change was analysed under the third body hypothesis and it is superimposed on a parabolic variation, which may be attributed to either mass transfer from the less-massive component to the more-massive one, or an additional component bonded to the double binary system with a longer period. In the last spectroscopic study Barden (1987) succeeded in obtaining the spectra of the previously suspected third companion in the binary XY Leo. For this W-type eclipsing system, one can estimate the spectroscopic mass-ratio of the components based on the semiamplitudes of the velocity curves $\left(K_{\mathrm{c}}=124.1\right.$ and $K_{\mathrm{h}}=$ $204.7 \mathrm{~km} \mathrm{~s}^{-1}$ for the more- and less-massive components, respectively) as $q=m_{\mathrm{c}} / m_{\mathrm{h}} \sim 1.64$. Moreover, Barden (1987) revealed the binary nature of the third body and obtained the radial velocities of the third and fourth components successfully. Based on this study, he found that the companion of XY Leo is a BY Dra binary system, composed of late-K to mid-M dwarf stars with an orbital period of 0.805 days. Also, Barden (1987) showed that a significant portion of the chromospheric emission observed by many investigators from XY Leo was, instead, associated with the components of the BY Dra binary, which together with XY Leo forms a quadruple system. 
Table 1. Absolute parameters for XY Leo (Yakut et al. 2003).

\begin{tabular}{lll}
\hline Parameter & Large star & Small star \\
\hline Mass $\left[\mathrm{M}_{\odot}\right]$ & $0.82(2)$ & $0.50(1)$ \\
Radius $\left[\mathrm{R}_{\odot}\right]$ & $0.85(2)$ & $0.68(2)$ \\
Effective temperature $[\mathrm{K}]$ & $4524(14)$ & 4850 \\
Luminosity $\left[\mathrm{L}_{\odot}\right]$ & 0.267 & 0.226 \\
$M_{\text {bol }}$ & 6.2 & 6.4 \\
\hline
\end{tabular}

Yakut et al. (2003) analyzed the B and V light curves obtained during 2000-2001 with the $48 \mathrm{~cm}$ Cassegrain telescope at Ege University Observatory. They applied Wilson-Devinney code by assuming that there was a third light in the system. They found the orbit inclination of $67^{\circ} .6$, temperature of the cooler component $T_{\mathrm{c}} \sim 4524 \mathrm{~K}$, shallow contact degree $\left(f_{\text {over }} \sim 6.7 \%\right)$ and the third light contribution of about $2 \%$ in the $B$ - and $6 \%$ in the $V$-light curve. By combining the photometric solution with spectroscopic elements given by Barden (1987), they estimated the absolute parameters of the system, which are given in Table 1.

\subsection{EE Cet}

Variability of the combined light of the visual binary ADS 2163 was discovered by the Hipparcos satellite ESA (1997). The Hipparcos photometric observations of the system show a light-curve variation with an amplitude of $0.23 \mathrm{mag}$ and a 0.38 -day period. Rucinski et al. (2002) correctly found that the source of variability is the southern, fainter companion, which is classified as a W Ursa Majoris (W UMa) type eclipsing binary EE Cet $(P \sim 0.38$ days). In their radial velocity study EE Cet was observed without contamination by the northern companion, separated by 5.6". Proceeding from well defined radial velocity curves for both components in the EE Cet system, they determined mass ratio to be $q=m_{\mathrm{h}} / m_{\mathrm{c}}=0.315$. The indices (h,c) refer to the hotter less-massive and cooler more-massive component, respectively. Rucinski et al. (2002) list F8 V to be the spectral type of the system and that it belongs to the $\mathrm{W}$ subclass of W UMa configuration.

\section{$1.3 A Q P S C$}

The eclipsing binary system AQ Psc $(P \sim 0.476$ days $)$ was discovered by Sarma \& Radhakrishnan (1982) as a W UMa variable with a period of 0.47564 days. From $B-V=+0^{m} .5$ and $U-B=+0^{m} .0$ at maximum light, they estimated the spectral type of the system to be F8 V. Lu \& Rucinski (1999) observed the radial velocities of AQ Psc and determined its orbital elements. Proceeding from the well defined radial velocity curves for both components, they determined mass ratio of this A-type system to be $q=m_{\mathrm{c}} / m_{\mathrm{h}}=0.226$. The indices (h,c) refer to the hotter (more-massive) primary and cooler (lessmassive) secondary component, respectively. The first
Table 2. Absolute parameters for AQ Psc (Yamasaki 2005).

\begin{tabular}{lll}
\hline Parameter & Large star & Small star \\
\hline Mass $\left[\mathrm{M}_{\odot}\right]$ & $1.69(4)$ & $0.38(1)$ \\
Radius $\left[\mathrm{R}_{\odot}\right]$ & $1.77(2)$ & $0.89(1)$ \\
Effective temperature $[\mathrm{K}]$ & 6250 & $6180(60)$ \\
Luminosity $\left[\mathrm{L}_{\odot}\right]^{\mathrm{A}}$ & 4.283 & 1.035 \\
$M_{\text {bol }}$ & 3.2 & 4.7 \\
\hline
\end{tabular}

${ }^{\text {A }}$ Luminosities and $M_{\text {bol }}$ are estimated from the radii and temperatures by $L / L_{\odot}=\left(R / R_{\odot}\right)^{2}(T / 5780)^{4}$, assuming $M_{\text {bol }}^{\odot}=4.75$.

extensive photometric study of AQ Psc was made by Yamasaki (2005). Yamasaki analyzed the light-curves obtained between October 2003 and February 2004 by CCD photometry through $B V$ filters. Using their own code based on the Roche model Yamasaki (1981) estimated the basic system parameters, which are given in Table 2. Pribulla \& Rucinski (2006) conclude that most contact binary stars exist in multiple systems. AQ Psc has been indicated by the latter authors as a system in which we could expect the presence of the third component.

\section{New observations}

New photoelectric $B V$ light curves of three close eclipsing binaries XY Leo, EE Cet and AQ Psc were observed at the Rozhen National Astronomical Observatory, Bulgaria in the period October 2004-February 2005, by using the 0.6-m Cassegrain telescope, equipped with an $U B V$, single-channel, photon-counting, computer-controlled photometer. An integration time of 10 seconds was chosen. Observational data are available through CDS, Strasbourg, France. ${ }^{1}$

These observations are employed in modelling of these three close binary systems (CBs), aimed at deriving their physical parameters and at studying the nature of their activity.

\subsection{XY Leo}

New photoelectric $B V$ observations of $\mathrm{XY}$ Leo $(\mathrm{BD}+$ $\left.18^{\circ} 2307\right)$ were made on the nights of 6 and 7 February 2005. The stars $\mathrm{BD}+18^{\circ} 2306(V=9.68, B=10.28)$ and $\mathrm{BD}+18^{\circ} 2305$ were chosen as a comparison and a check star, respectively. The same stars were used in most of the previous studies of the system either as a comparison or as a check star. The relevant catalogue data for $\mathrm{XY}$ Leo, comparison and check star, were extracted from SIMBAD database. A total of 467 observations were secured in $B$ and $V$ passbands. The differential observations, variable minus comparison, were corrected for atmospheric extinction using the passband extinction coefficients obtained for each night from the

\footnotetext{
${ }^{1}$ http://cdsweb.u-strasbg.fr/
} 
brightness variation of the comparison star. The standard deviations ( $1 \sigma$ error) of the data points were estimated as $\sigma_{B}=0.012$ and $\sigma_{V}=0.014$.

The observations cover two primary and two secondary minima. Their timings were calculated using the method of Kwee \& van Woerden (1956) and are listed in Table 3.

From estimated and previously published times of minima of the light curves, we have the following updated ephemeris for calculating the orbital phases:

$$
\operatorname{HJD} I_{\min }=2451884.4443(10)+0.2840975(3) \times E
$$

The light levels estimated by averaging data around the maxima and minima and their differences are listed in Table 4. The light curves are slightly asymmetric. There is also a noticeable difference between the levels of the two minima in $B$ and $V$ colours (see Figure 1 and Table 4$)$ and slight variations in the $\Delta(B-V)$ colour curve with orbital phase.

The shape of the light curve indicates the overcontact configuration of the system. Relatively small amplitudes of the light curves are the result of a low orbit inclination producing partial eclipses.

\subsection{EE Cet}

New observations of EE Cet $\left(\mathrm{BD}+08^{\circ} 433 B\right)$ in $B V$ were obtained during 7 and 8 October 2004. BD $+08^{\circ} 430$ and $\mathrm{BD}+07^{\circ} 431$ were chosen as a comparison and a check star, respectively. The relevant catalogue data for the stars were extracted from SIMBAD database. A total of 449 observations in $B$ and $V$ passbands were secured. The standard deviations ( $1 \sigma$ error) of the observations were estimated as $\sigma_{B, V}=0.008$.

The differential $B$ and $V$ light curves and $\Delta(B-V)$ colour curve are shown in Figure 2. The observations cover three minima. Their timings were calculated using the method of Kwee \& van Woerden (1956) and are listed in Table 3. By using these and previously published minima of the system we determined the following updated light elements:

$$
\operatorname{HJD} I_{\min }=2451818.7998(17)+0.3799215(5) \times E
$$

This ephemeris is used to compute the phase of the observations.

The light levels estimated by averaging data around the maxima and minima (by taking a $\Delta \phi= \pm 0.02$ interval) and their differences are listed in Table 4. The light curves are slightly asymmetric with the secondary maxima higher than the primary ones. There is also a noticeable difference between the levels of the two minima in $B$ and $V$ colours (see Figure 2 and Table 4) and slight variations in the $\Delta(B-V)$ colour curve around minima.

The shape of the light curve indicates the overcontact configuration of the system. However, the presence of the northern, brighter visual companion, about 5.6" from
Table 3. Times of minima for XY Leo, EE Cet and AQ Psc derived from our observations.

\begin{tabular}{lcccc}
\hline System & HJD 2400000+ & Error & Type & Filter \\
\hline XY Leo & 53408.3413 & 0.0005 & I & BV \\
& 53408.6248 & 0.0040 & I & BV \\
& 53408.4822 & 0.0004 & II & BV \\
& 53409.3351 & 0.0007 & II & BV \\
\hline EE Cet & 53286.4361 & 0.0003 & I & BV \\
& 53287.5770 & 0.0004 & I & BV \\
& 53287.3850 & 0.0003 & II & BV \\
\hline AQ Psc & 53327.4538 & 0.0005 & I & BV \\
& 53347.1932 & 0.0006 & II & BV \\
\hline
\end{tabular}

Table 4. The light levels and their differences in the

\begin{tabular}{|c|c|c|}
\hline XY Leo & $\Delta B$ & $\Delta V$ \\
\hline Max. light at 0.25 & $0.206 \pm 0.005$ & $-0.080 \pm 0.004$ \\
\hline Max. light at 0.75 & $0.194 \pm 0.005$ & $-0.084 \pm 0.004$ \\
\hline Min. light at 0.00 & $0.684 \pm 0.006$ & $0.346 \pm 0.006$ \\
\hline Min. light at 0.50 & $0.580 \pm 0.005$ & $0.245 \pm 0.005$ \\
\hline$\Delta \max \left(m_{0.25}-m_{0.75}\right)$ & 0.012 & 0.004 \\
\hline$\Delta \min \left(m_{0.00}-m_{0.50}\right)$ & 0.104 & 0.101 \\
\hline$\Delta \max \left(m_{0.25}-m_{0.00}\right)$ & -0.478 & -0.426 \\
\hline$\Delta \max \left(m_{0.25}-m_{0.50}\right)$ & -0.374 & -0.325 \\
\hline EE Cet & $\Delta B$ & $\Delta V$ \\
\hline Max. light at 0.25 & $0.013 \pm 0.005$ & $-0.028 \pm 0.012$ \\
\hline Max. light at 0.75 & $0.002 \pm 0.010$ & $-0.039 \pm 0.013$ \\
\hline Min. light at 0.00 & $0.249 \pm 0.003$ & $0.201 \pm 0.005$ \\
\hline Min. light at 0.50 & $0.238 \pm 0.006$ & $0.186 \pm 0.008$ \\
\hline$\Delta \max \left(m_{0.25}-m_{0.75}\right)$ & 0.011 & 0.011 \\
\hline$\Delta \min \left(m_{0.00}-m_{0.50}\right)$ & 0.011 & 0.015 \\
\hline$\Delta \max \left(m_{0.25}-m_{0.00}\right)$ & -0.236 & -0.229 \\
\hline$\Delta \max \left(m_{0.25}-m_{0.50}\right)$ & -0.225 & -0.214 \\
\hline AQ Psc & $\Delta B$ & $\Delta V$ \\
\hline Max. light at 0.25 & $9.010 \pm 0.005$ & $8.565 \pm 0.004$ \\
\hline Max. light at 0.75 & $9.012 \pm 0.005$ & $8.567 \pm 0.004$ \\
\hline Min. light at 0.00 & $9.373 \pm 0.006$ & $8.920 \pm 0.006$ \\
\hline Min. light at 0.50 & $9.354 \pm 0.005$ & $8.902 \pm 0.005$ \\
\hline$\Delta \max \left(m_{0.25}-m_{0.75}\right)$ & -0.002 & -0.002 \\
\hline$\Delta \min \left(m_{0.00}-m_{0.50}\right)$ & 0.019 & 0.018 \\
\hline$\Delta \max \left(m_{0.25}-m_{0.00}\right)$ & -0.363 & -0.355 \\
\hline$\Delta \max \left(m_{0.25}-m_{0.50}\right)$ & -0.344 & -0.337 \\
\hline
\end{tabular}
$B$ and $V$ curves of XY Leo, EE Cet and AQ Psc.

EE Cet, complicates photometric observations and introduces additional complications in their interpretation. Due to the close proximity of the visual companion it could not be excluded from the diaphragm during the observations and, therefore, our photometric measurements were contaminated by the third light. Relatively low amplitudes of the light curves are the result of a 


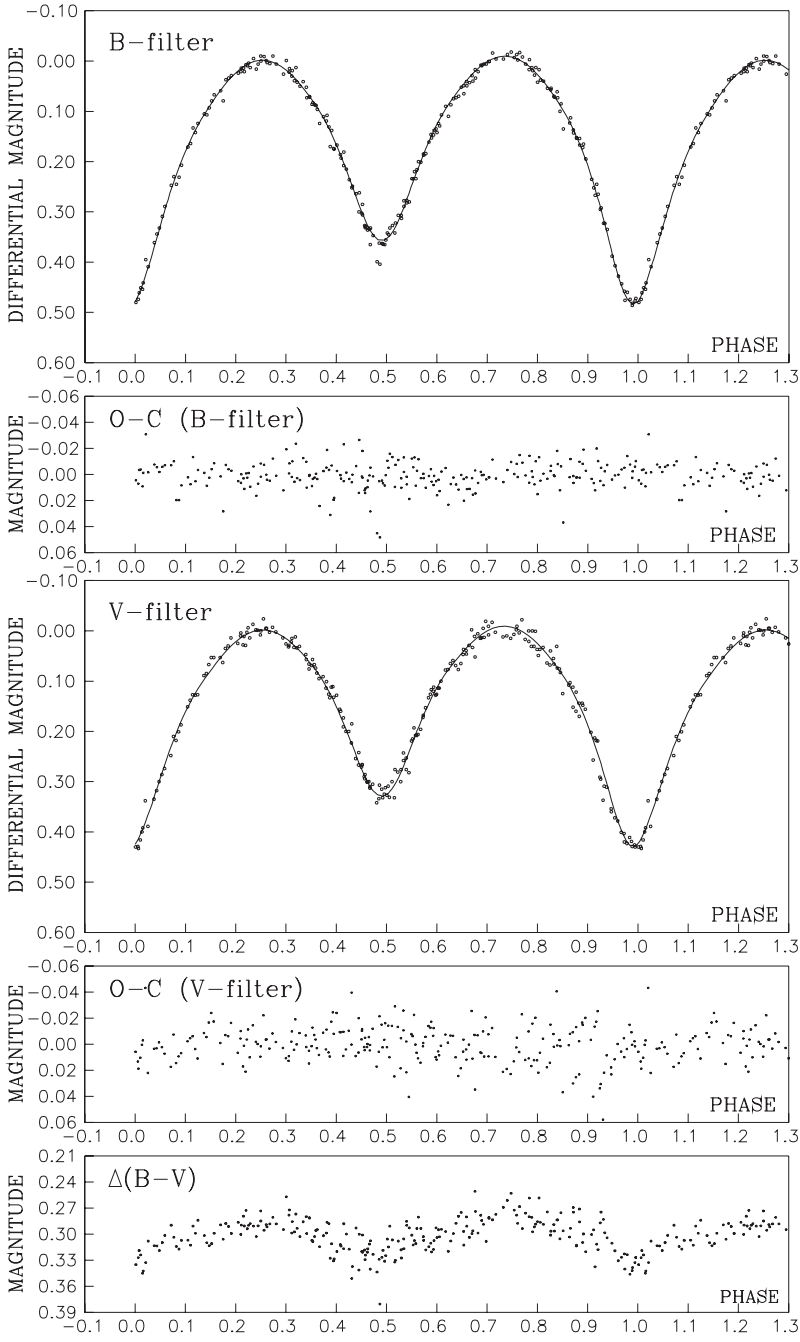

Figure 1 Observed (dots) and final synthetic (solid line) lightcurves of $\mathrm{XY}$ Leo with final $\mathrm{O}-\mathrm{C}$ residuals obtained by simultaneous analysis of the $B$ and $V$ observations. Differential $\Delta(B-V)$ colour curve is given at the bottom.

substantial contribution of the third light to the total light of the system. Namely, the third-light solution gives the orbit inclination of $78^{\circ} .5$ and high overcontact degree $\left(f_{\text {over }} \sim 33 \%\right)$, producing partial eclipses.

\section{$2.3 A Q P s C$}

New photoelectric observations of AQ Psc (BD+06 203) in $B V$ were made over six nights (24 October, 17 and 20 November, 7 December 2004, and 02 January and 11 February 2005).

$\mathrm{BD}+06^{\circ} 197(V=8.42, B=9.33)$ and $\mathrm{BD}+06^{\circ} 200$ were chosen as comparison and check star, respectively. The relevant catalogue data for the stars were extracted from the SIMBAD database. A total of 478 observations were secured in $B$ and $V$ passbands. The standard deviations ( $1 \sigma$ error) of the data points were estimated as $\sigma_{B}=0.009$ and $\sigma_{V}=0.01$. The $B$ and $V$ light curves and $B-V$ colour curve are shown in Figure 3.

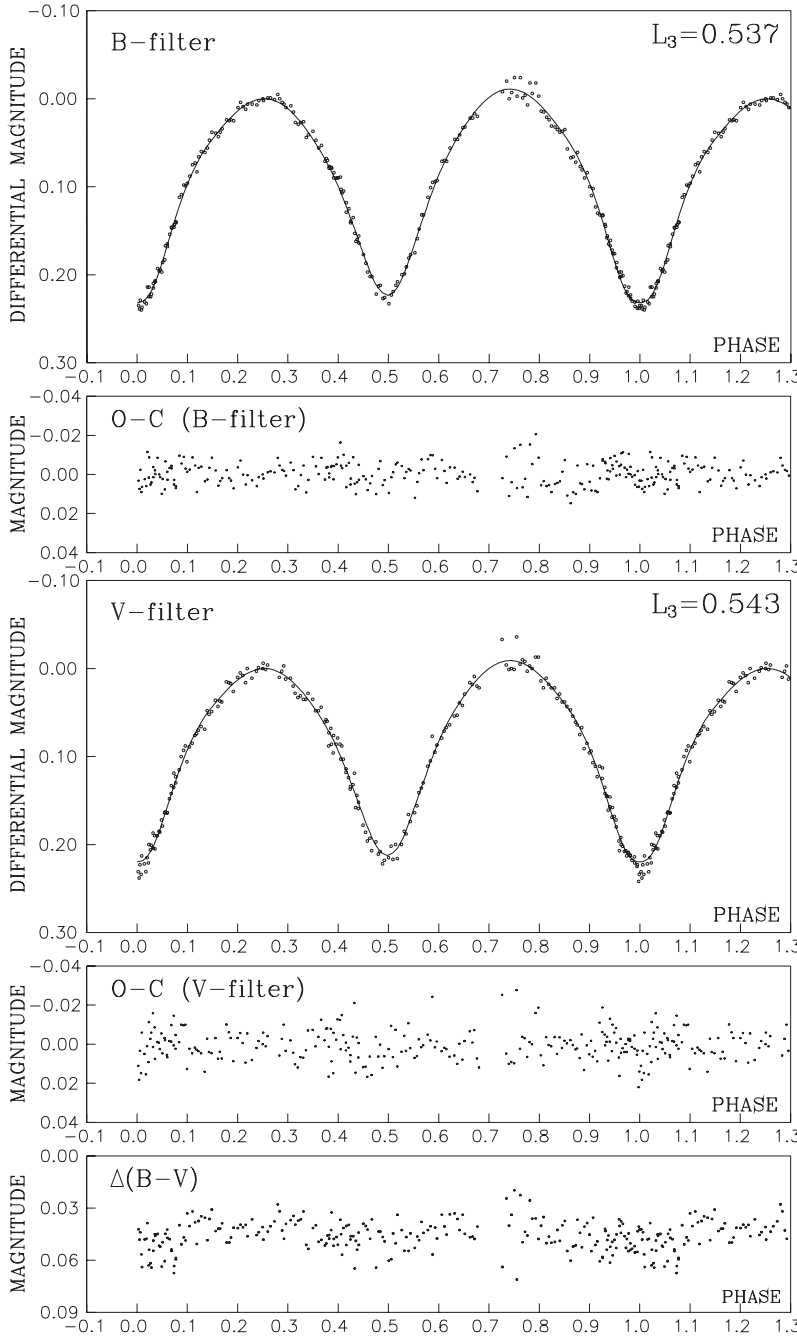

Figure 2 Observed (dots) and final synthetic (solid line) lightcurves of EE Cet with final $\mathrm{O}-\mathrm{C}$ residuals obtained with third light effect $L_{3} \sim 0.54$. Differential $\Delta(B-V)$ colour curve is given at the bottom.

The observations cover two minima. Their timings were calculated using the method of Kwee \& van Woerden (1956) and are listed in Table 3.

By using these and previously published minima of the system we determined the following updated ephemeris employed in computing the phase of observations:

$$
\operatorname{HJD} I_{\min }=2449283.3254(11)+0.4756119(2) \times E
$$

The light levels estimated by averaging data around the maxima and minima (by taking a $\Delta \phi= \pm 0.02$ interval) and their differences are listed in Table 4. The light curves are slightly asymmetric with the primary maxima higher than the secondary ones. There is also a noticeable difference between the levels of the two minima in $B$ and $V$ colours (see Figure 3 and Table 4 ) and slight variations in the $B-V$ colour curve with orbital phase.

The shape of the light curve indicates the overcontact configuration of the system. Relatively small amplitudes 


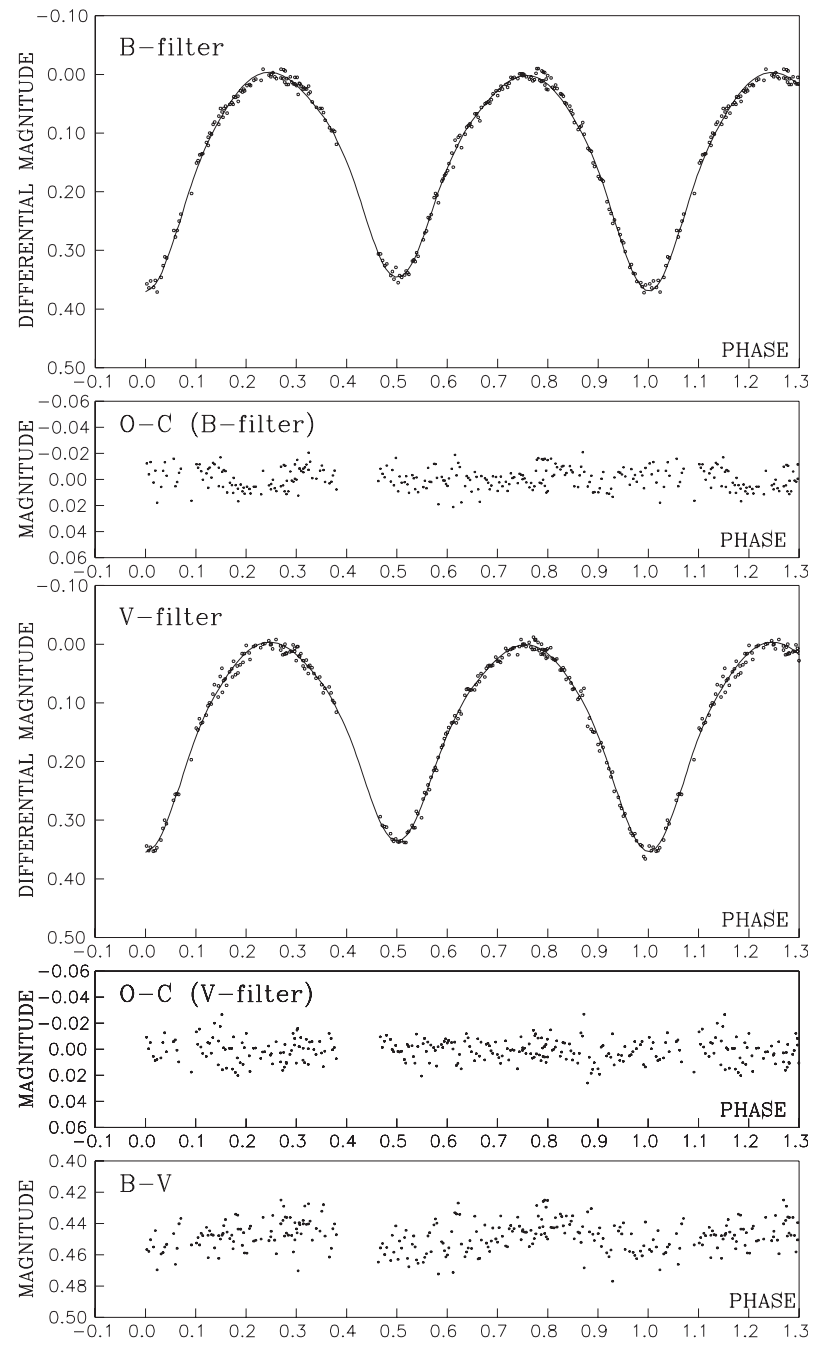

Figure 3 Observed (dots) and final synthetic (solid line) lightcurves of AQ Psc with final $\mathrm{O}-\mathrm{C}$ residuals obtained by simultaneous analysis of the $B$ and $V$ observations. $B-V$ colour curve is given at the bottom.

of the light curves are the result of a low orbit inclination, producing partial eclipses.

\section{The results of the light-curve analyses}

In order to model the $B$ and $V$ band light curves of these binary systems we used the code developed by Djurašević (1992a), modified for overcontact configuration by Djurašević et al. (1998). The code is based on the Roche model and the principles given in Wilson \& Devinney (1971). The light-curve analyses were made by applying the inverse-problem method by Djurašević (1992b), based on the modified Marquardt (1963) algorithm. More details about the code and the method of light-curve analysis can be found in e.g. Djurašević et al. (2004a).

For the values of the stellar gravity-darkening exponents we used the theoretical prediction $\beta_{\mathrm{h}, \mathrm{c}}=0.08$, given by Lucy (1967) for the stars with a convective envelope, in agreement with the spectral types of the components of the systems in this study. Consequently, for stellar albedos the values $A_{\mathrm{h}, \mathrm{c}}=0.5$ were used.

In agreement with Claret (2000), we chose a new nonlinear approximation for the limb-darkening law that can be written as:

$$
\begin{aligned}
I(\mu) / I(1)= & 1-a_{1}\left(1-\mu^{1 / 2}\right)-a_{2}(1-\mu) \\
& -a_{3}\left(1-\mu^{3 / 2}\right)-a_{4}\left(1-\mu^{2}\right)
\end{aligned}
$$

where $a_{1,2,3,4}$ are the limb-darkening passband specific coefficients, $I(1)$ is the passband-specific intensity at the center of the stellar disc and $\mu=\cos \gamma$, where $\gamma$ is the angle between the line of sight and the emergent flux.

The present light-curve analyses were carried out by the simultaneous solution of the light curves in $B$ and $V$, using a simple black-body approximation.

The estimated errors of the parameters arise from the non-linear least-squares method that the inverseproblem method is based on. The real uncertainties of these parameters may be approximately 2-3 times larger than the estimated ones. This is due to the influence of the errors of the model input parameters, which are taken as fixed in the inverse-problem solution.

The optimum fit of each passband-observed light curve (LCO) to the synthetic ones (LCC) are shown in Figures 1-3 for XY Leo, EE Cet and AQ Psc, respectively. The final $\mathrm{O}-\mathrm{C}$ residuals between the observed (LCO) and optimum synthetic (LCC) light curves are also given. Finally, the bottom panel in these figures shows the variation in the corresponding colour curve against orbital phase.

\section{$3.1 X Y$ Leo}

Since the results of previous light curve analyses were available to us, in the present light-curve analysis the mass ratio of the components was fixed at the value $q=m_{\mathrm{c}} / m_{\mathrm{h}}=1.64$, estimated by Yakut et al. (2003) who used the semi-amplitudes of velocity curves derived by Barden (1987) from radial velocity studies. According to Yakut et al. (2003), the temperature of the less-massive hotter component (eclipsed at $I_{\text {min }}$ ) was set at $T_{\mathrm{h}}=$ $4850 \mathrm{~K}$ in agreement with the $B-V$ colour of 0.82 and Popper (1980) calibration.

The light curves were analysed by assuming that there was a third-light in the system. According to Yakut et al. (2003) initial value of the third light $L_{3}$ was taken to be $0.019[B]$ and $0.059[V]$.

The light curves of XY Leo are slightly asymmetric. This tells us that the components of this contact binary exhibit activity. In order to describe all the features of the observed light curves we have tested several different hypotheses. Our very first assumption was a Roche model with no spots on the components. The deviations of the fit from the observed light curves, i.e. the $\mathrm{O}-\mathrm{C}$ residuals (especially in the phase interval $0.0-0.3$ ) point out the existence of certain activity on 
the system components which deforms the light curves making them slightly asymmetric.

Therefore, our next step was to treat stellar albedos as free parameters in the inverse-problem. This procedure provides a better fit to the observations and shows that the albedo of the secondary (hotter) component has to be much less than the expected value for stars with convective envelope. This suggests that we have a cool spot area on the secondary, located on the hemisphere facing the primary component. Finally, we fixed the albedos at their expected theoretical values, $A_{\mathrm{h}, \mathrm{c}}=0.5$, appropriate for stars with convective envelopes, and introduced a cool spot area as a possible natural explanation for the behavior of the light curves. The cool spot region may be a consequence of the magnetic dynamo mechanism, i.e. solar-type activity.

In our code the active region is approximated by a circular spot, characterized by the temperature contrast of the spot with respect to the surrounding photosphere $\left(A_{\mathrm{s}}=T_{\mathrm{s}} / T_{\mathrm{h}}\right)$, the angular dimensions (radius) of the spot $\left(\theta_{\mathrm{s}}\right)$, and by the longitude $\left(\lambda_{\mathrm{s}}\right)$ and latitude $\left(\varphi_{\mathrm{s}}\right)$ of the spot center. The longitude $\left(\lambda_{\mathrm{s}}\right)$ is measured clockwise (as viewed from the direction of the $+z$-axis) from the $+x$-axis (line connecting the star centers) in the range $0^{\circ}-360^{\circ}$. The latitude $\left(\varphi_{\mathrm{s}}\right)$ is measured from $0^{\circ}$ at the stellar equator (orbital plane) to $+90^{\circ}$ towards the 'North' $(+z)$ pole and $-90^{\circ}$ towards the 'South' $(-z)$ pole.

The solutions of our simultaneous light-curves analysis of XY Leo (with and without cool spot) are given in Table 5. The first three rows of the table present the total number $n$ of $B$ and $V$ observations, the final sum of the squares of the residuals between observed (LCO) and synthetic (LCC) light curves

$$
\sum_{i=1}^{n}\left(O_{i}-C_{i}\right)^{2}
$$

and the root-mean-square of the residuals

$$
\sigma_{r m s}=\sqrt{\frac{\sum_{i=1}^{n}\left(O_{i}-C_{i}\right)^{2}}{(n-1)}}
$$

In the same table we give the spot characteristics (spot temperature factor $A_{\mathrm{s}}=T_{\mathrm{s}} / T_{\mathrm{h}}$, angular radius $\theta_{\mathrm{s}}$, longitude $\lambda_{\mathrm{s}}$ and latitude $\varphi_{\mathrm{s}}$ ). These parameters are determined by a simultaneous fitting of the light curves in $B$ and $V$ photometric bands.

The inclination of the orbit was estimated to be $i \sim 68^{\circ}$. A relatively low amplitude of the light curves thus may be explained by partial eclipses. The obtained solution shows the marginal degree of overcontact $\left(f_{\text {over }} \sim 2.4 \%\right)$ with a relatively large temperature difference between the components $\left(\Delta T=T_{\mathrm{h}}-T_{\mathrm{c}} \sim 330 \mathrm{~K}\right)$.

From Table 5 and Figure 1 it is evident that the Roche model with cool spot area on the less-massive star gives a satisfactory fit to the analysed light curves. The spot temperature is about $23 \%$ lower than in the surrounding photosphere. The spot at longitude $\lambda_{\mathrm{s}} \sim 10^{\circ}$ produces a
Table 5. Results of the simultaneous analyses of the $\mathrm{XY}$ Leo $B$ and $V$ light-curves obtained by solving the inverse problem for the Roche model with and without the cool spot area on the less-massive (hotter) component.

\begin{tabular}{lll}
\hline Quantity & with spot & without spot \\
\hline$n$ & 468 & 468 \\
$\Sigma(\mathrm{O}-\mathrm{C})^{2}$ & 0.0767 & 0.1048 \\
$\sigma_{\mathrm{rms}}$ & 0.0128 & 0.0150 \\
$q=m_{\mathrm{c}} / m_{\mathrm{h}}$ & 1.64 & 1.64 \\
$T_{\mathrm{h}}$ & 4850 & 4850 \\
$A_{\mathrm{h}}=A_{\mathrm{c}}$ & 0.5 & 0.5 \\
$\beta_{\mathrm{h}}=\beta_{\mathrm{c}}$ & 0.08 & 0.08 \\
$f_{\mathrm{h}}=f_{\mathrm{c}}$ & 1.0 & 1.0 \\
$A_{\mathrm{s}}=T_{\mathrm{s}} / T_{\mathrm{h}}$ & $0.77 \pm 0.05$ & \\
$\theta_{\mathrm{s}}$ & $40.1 \pm 0.9$ & \\
$\lambda_{\mathrm{s}}$ & $9.9 \pm 1.2$ & \\
$\varphi_{\mathrm{s}}$ & $-45.3 \pm 1.5$ & \\
$T_{\mathrm{c}}$ & $4524 \pm 10$ & $4486 \pm 13$ \\
$F_{\mathrm{h}}$ & $1.004 \pm 0.001$ & $1.007 \pm 0.001$ \\
$i\left[{ }^{\circ}\right]$ & $68.2 \pm 0.1$ & $67.5 \pm 0.2$ \\
$L_{3}(\mathrm{~B})$ & $0.021 \pm 0.004$ & $0.020 \pm 0.005$ \\
$L_{3}(\mathrm{~V})$ & $0.061 \pm 0.005$ & $0.060 \pm 0.006$ \\
$\Omega_{\mathrm{h}, \mathrm{c}}$ & $4.719 \pm 0.003$ & $4.711 \pm 0.003$ \\
$\Omega_{\text {in }}, \Omega_{\text {out }}$ & $4.7333,4.1493$ & \\
$f_{\text {over }}[\%]$ & 2.44 & 3.87 \\
$R_{\mathrm{h}, \mathrm{c}}[D=1]$ & $0.317,0.399$ & $0.318,0.400$ \\
$L_{\mathrm{h}} /\left(L_{\mathrm{h}}+L_{\mathrm{c}}+L_{3}\right)$ & $0.464[\mathrm{~B}] ;$ & $0.507[\mathrm{~B}] ;$ \\
& $0.436[\mathrm{~V}]$ & $0.474[\mathrm{~V}]$ \\
\hline$N_{0}$ &
\end{tabular}

Note: $n$ : total number of the $B$ and $V$ observations, $\Sigma(\mathrm{O}-\mathrm{C})^{2}$ : final sum of squares of residuals between observed (LCO) and synthetic (LCC) light-curves, $\sigma_{\text {rms }}$ : root-mean-square of the residuals, $q=m_{\mathrm{c}} / m_{\mathrm{h}}$ : mass ratio of the components, $T_{\mathrm{h}, \mathrm{c}}$ : temperature of the hotter primary and cooler secondary, $\beta_{\mathrm{h}, \mathrm{c}}$ : $A_{\mathrm{h}, \mathrm{c}}, f_{\mathrm{h}, \mathrm{c}}$ : gravity-darkening exponents, albedos and nonsynchronous rotation coefficients of the components respectively, $A_{\mathrm{s}}, \theta_{\mathrm{s}}, \lambda_{\mathrm{s}}$ and $\varphi_{\mathrm{s}}$ : spot temperature coefficient, angular dimension, longitude and latitude (in arc degrees), $F_{\mathrm{h}}$ : filling factor for the critical Roche lobe of the hotter primary, $i\left[^{\circ}\right]$ : orbit inclination (in arc degrees), $L_{3}$ : third light, $\Omega_{\mathrm{h}, \mathrm{c}}, \Omega_{\mathrm{in}}, \Omega_{\mathrm{out}}$ : dimensionless surface potentials of the components and of the inner and outer contact surfaces respectively, $f_{\text {over }}[\%]$ : degree of overcontact, $R_{\mathrm{h}, \mathrm{c}}$ : polar radii of the components in units of the distance between the component centres and $L_{\mathrm{h}} /\left(L_{\mathrm{h}}+L_{\mathrm{c}}+\right.$ $\left.L_{3}\right)$ : luminosity $(B ; V)$ of the less-massive hotter star (including cool spot).

slight light-curve asymmetry (especially evident in the phase interval $0.0-0.3$ ). The noticeable phase dependence of the colour curve $\Delta(B-V)$ (Figure 1) is mainly caused by the component eclipses, but its form is, at least partially, affected by the presence of the spot region.

\subsection{EE Cet}

In the light-curve analysis the mass ratio of the components was fixed at $q=m_{\mathrm{h}} / m_{\mathrm{c}}=0.315$, estimated by Rucinski et al. (2002) from their radial velocity study. 
Bearing in mind the spectral type $\mathrm{F} 8 \mathrm{~V}$, the temperature of the more-massive component was set at $T_{\mathrm{c}}=6095 \mathrm{~K}$ in agreement with the calibration of Popper (1980).

In the light-curve synthesis we have included the third light effect. This effect can significantly change the shape of the light curve, see e.g. Figure 2 in Djurašević et al. (2004b). The third light evidently changes the light-curve amplitude. This is reflected, above all, in the estimates of the orbit inclination and in the overcontact degree of the system. Therefore, full appreciation of this effect, i.e. an accurate estimate of the parameter $L_{3}$ related to the effect is necessary in order to obtain valid estimates of the physical parameters of the system. We have to point out that the third light contribution to the total light cannot be estimated by solving the inverse problem, i.e. by light-curve analysis only. So this has to be done in an independent way.

We estimated the third light parameter $L_{3} \sim 0.582$ in the $V$ band from the magnitudes of the visual companions of ADS 2163 given by Lampens et al. (2001). We used Tycho-2 catalogue by Høg et al. (2000) as another source. Apparent magnitudes from this catalogue for ADS 2163, transformed to $U B V$ system, are $V=9.620, B=10.094$ and $B-V=0.474$ for EE Cet, and $V=9.458, B=9.905$ and $B-V=0.447$ for the visual companion. From these data we obtain $L_{3} \sim 0.537$ in $B$ band, and $L_{3} \sim 0.543$ in $V$ band. Other sources give $L_{3} \sim 0.517$ from the Hipparcos ESA (1997) catalogue and $L_{3} \sim 0.569$ from the second edition of the CCDM catalogue by Dommanget \& Nys (2002). With this significant discrepancy in the estimates of the third light contribution, we adopted $L_{3} \sim 0.537$ for $B$, and $L_{3} \sim 0.543$ for the $V$ filter, estimated by using the Tycho-2 catalogue. The Hipparcos and $C C D M$ catalogue $L_{3}$-estimates, if averaged, give approximately the same value.

The light curves of EE Cet have a peculiar shape. We have assumed that the secondary, less-massive component is eclipsed in the deeper (primary) minimum (W-type contact system). The obtained synthetic light curves, with no active regions, do not fit the observations well. The deviations of the fit from the observed light curves, indicate the existence of certain activity on the system components, which deforms the light curves making them slightly asymmetric. Therefore, our next step was to treat stellar albedos as free parameters in the inverse problem solution. This procedure provided better fits to the observations. The model with no spots show that the primary component's albedo is lesser than the theoretical prediction (for stars with a convective envelope), which indicates a possible cool spot on the primary, at the hemisphere facing the secondary star. Finally, we fixed the albedos at their expected theoretical values, $A_{\mathrm{c}, \mathrm{h}}=0.5$, appropriate for stars with a convective envelope, and solved the inverse problem by optimizing the system and cool-spot parameters.

Simultaneous optimization of the system and coolspot parameters provides a very good fit to the observed light curves. Relatively low amplitudes of the light curves are the result of the substantial contribution of the third light to the total light of the system $\left(L_{3} \sim 0.54\right)$.

The inclination of the orbit was estimated to be $i \sim 79^{\circ}$. The filling coefficients for the critical Roche lobe $\left(F_{\mathrm{c}} \sim 1.03\right)$ indicate a configuration with high degree of overcontact ( $f_{\text {over }} \sim 33 \%$ ).

From Table 6 and Figure 2 is evident that the Roche model with cool spot area on the more-massive cooler star gives a satisfactory fit to the analysed light curves.

\section{$3.3 A Q P S C$}

In the light-curve analysis the mass ratio of the components was fixed at the value $q=m_{\mathrm{c}} / m_{\mathrm{h}}=0.226$, estimated by Lu \& Rucinski (1999) from their radial velocity study. According to Sarma \& Radhakrishnan (1982), the spectral type of the system is F8 V. Consequently, the temperature of the more-massive component was set at $T_{\mathrm{h}}=6095 \mathrm{~K}$ in agreement with spectral type and Popper (1980) calibration.

In order to describe all the features seen in the observed light curves of AQ Psc we have tested several different hypotheses. The first assumption was Roche model with no spots on the components. We assumed that primary, more-massive component is eclipsed in the deeper (primary) minimum (A-type contact system). Solving the inverse problem, we obtained the overcontact configuration with similar temperatures of the components $\left(\Delta T=T_{\mathrm{h}}-T_{\mathrm{c}} \sim 150 \mathrm{~K}\right)$. Having in mind a considerable difference in their masses $(q=0.226)$, it is reasonable to suppose that there is some energy exchange between the components, equalising the temperature in the common envelope. Therefore, our next step was to treat stellar albedos as free parameters in the inverse problem solution. This procedure provides better fit to the observations, but it shows that the albedo of the secondary (cooler) component has to be much larger than the expected value for the stars with convective envelope. This suggests that on the secondary, opposite to the Lagrangean point $L_{1}$ in the neck region, we have a bright area with an increased temperature. So, finally we fixed albedos at their expected theoretical values, $A_{\mathrm{h}, \mathrm{c}}=0.5$, appropriate for stars with convective envelope, and introduced a bright spot area as a possible natural explanation for the behavior of the light curve. The bright spot region can be explained as a consequence of an intensive energy exchange between the components through the neck of the common envelope, i.e. of the mechanism equilibrating stellar temperatures.

The inclination of the orbit was estimated to be $i \sim 68.9^{\circ}$. A relatively small amplitude of the light curves thus may be explained by partial eclipses. The filling coefficient for the critical Roche lobe $\left(F_{\mathrm{h}}\right)$ indicates a configuration with relatively high degree of overcontact $\left(f_{\text {over }} \sim 23 \%\right)$.

From the solutions obtained we can see that the secondary is the cooler component of the system, and 
Table 6. Results of the simultaneous $B$ and $V$ light curve analyses of the W-type system EE Cet, obtained by solving the inverse problem for the Roche model with and without the cool spot area on the more-massive (cooler) component.

\begin{tabular}{lll}
\hline Quantity & with spot & without spot \\
\hline$n$ & 449 & 449 \\
$\Sigma(\mathrm{O}-\mathrm{C})^{2}$ & 0.0228 & 0.0286 \\
$\sigma_{\mathrm{rms}}$ & 0.0071 & 0.0080 \\
$q=m_{2} / m_{1}$ & 0.315 & 0.315 \\
$\mathrm{~L}_{3}$ & $0.537[\mathrm{~B}]$, & $0.537[\mathrm{~B}]$, \\
& $0.543[\mathrm{~V}]$ & $0.543[\mathrm{~V}]$ \\
$T_{\mathrm{c}}$ & 6095 & 6095 \\
$\beta_{\mathrm{h}}=\beta_{\mathrm{c}}$ & 0.08 & 0.08 \\
$A_{\mathrm{h}}=A_{\mathrm{c}}$ & 0.5 & 0.5 \\
$f_{\mathrm{h}}=f_{\mathrm{c}}$ & 1.0 & 1.0 \\
$A_{\mathrm{s}}=T_{\mathrm{s}} / T_{\mathrm{c}}$ & $0.91 \pm 0.01$ & \\
$\theta_{\mathrm{s}}$ & $15.8 \pm 0.6$ & \\
$\lambda_{\mathrm{s}}$ & $301.8 \pm 4.2$ & \\
$\varphi_{\mathrm{s}}$ & $21.5 \pm 7.0$ & \\
$T_{\mathrm{h}}$ & $6314 \pm 12$ & $6253 \pm 14$ \\
$F_{\mathrm{c}}$ & $1.029 \pm 0.001$ & $1.029 \pm 0.001$ \\
$i\left[{ }^{\circ}\right]$ & $78.5 \pm 0.2$ & $79.1 \pm 0.3$ \\
$\Omega_{\mathrm{c}, \mathrm{h}}$ & $2.435 \pm 0.002$ & $2.435 \pm 0.002$ \\
$\Omega_{\mathrm{in}}, \Omega_{\text {out }}$ & $2.4991,2.3034$ & $2.4991,2.3034$ \\
$f_{\mathrm{over}}[\%]$ & 32.69 & 32.81 \\
$R_{\mathrm{c}}, R_{\mathrm{h}}[\mathrm{D}=1]$ & $0.465,0.279$ & $0.465,0.279$ \\
$L_{\mathrm{c}} /\left(L_{\mathrm{c}}+L_{\mathrm{h}}+L_{3}\right)$ & $0.319[\mathrm{~B}] ;$ & $0.325[\mathrm{~B}] ;$ \\
\hline${ }$ & $0.317[\mathrm{~V}]$ & $0.322[\mathrm{~V}]$ \\
\hline & &
\end{tabular}

Note: $n$ : total number of the $B$ and $V$ observations, $\Sigma(\mathrm{O}-\mathrm{C})^{2}$ : final sum of squares of residuals between observed (LCO) and synthetic (LCC) light-curves, $\sigma_{\text {rms }}$ : root-mean-square of the residuals, $q=m_{\mathrm{c}} / m_{\mathrm{h}}$ : mass ratio of the components, $T_{\mathrm{h}, \mathrm{c}}$ : temperature of the hotter primary and cooler secondary, $\beta_{\mathrm{h}, \mathrm{c}}$ : $A_{\mathrm{h}, \mathrm{c}}, f_{\mathrm{h}, \mathrm{c}}$ : gravity-darkening exponents, albedos and nonsynchronous rotation coefficients of the components respectively, $A_{\mathrm{s}}, \theta_{\mathrm{s}}, \lambda_{\mathrm{s}}$ and $\varphi_{\mathrm{s}}:$ spot temperature coefficient, angular dimension, longitude and latitude (in arc degrees), $F_{\mathrm{h}}$ : filling factor for the critical Roche lobe of the hotter primary, $i\left[^{\circ}\right]$ : orbit inclination (in arc degrees), $L_{3}$ : third light, $\Omega_{\mathrm{h}, \mathrm{c}}, \Omega_{\mathrm{in}}, \Omega_{\mathrm{out}}$ : dimensionless surface potentials of the components and of the inner and outer contact surfaces respectively, $f_{\text {over }}[\%]$ : degree of overcontact, $R_{\mathrm{h}, \mathrm{c}}$ : polar radii of the components in units of the distance between the component centres and $L_{\mathrm{h}} /\left(L_{\mathrm{h}}+L_{\mathrm{c}}+\right.$ $\left.L_{3}\right)$ : luminosity $(B ; V)$ of the less-massive hotter star (including cool spot).

that the mean gravity of the secondary is somewhat smaller than that of the primary, which holds well for A-type W UMa systems.

From Table 7 and Figure 3 it is evident that the Roche model with bright spot area on the less-massive star, near the neck region between the components gives a satisfactory fit to the analysed light curves. In this region, which is probably a consequence of the energy exchange between the components, the temperature is increased by about $6 \%$ compared to the surrounding photosphere. The
Table 7. Results of the simultaneous analyses of the AQ Psc $B$ and $V$ light-curves obtained by solving the inverse problem for the Roche model with and without the bright spot area in the neck region on the lessmassive (cooler) component.

\begin{tabular}{|c|c|c|}
\hline Quantity & with spot & without spot \\
\hline$n$ & 478 & 478 \\
\hline$\Sigma(\mathrm{O}-\mathrm{C})^{2}$ & 0.0342 & 0.0444 \\
\hline$\sigma$ & 0.0084 & 0.0096 \\
\hline$q=m_{\mathrm{c}} / m_{\mathrm{h}}$ & 0.226 & 0.226 \\
\hline$T_{\mathrm{h}}$ & 6095 & 6095 \\
\hline$A_{\mathrm{h}}=A_{\mathrm{c}}$ & 0.5 & 0.5 \\
\hline$\beta_{\mathrm{h}}=\beta_{\mathrm{c}}$ & 0.08 & 0.08 \\
\hline$f_{\mathrm{h}}=f_{\mathrm{c}}$ & 1.0 & 1.0 \\
\hline$A_{\mathrm{bs}}=T_{\mathrm{bs}} / T_{\mathrm{c}}$ & $1.06 \pm 0.01$ & \\
\hline$\theta_{\mathrm{bs}}$ & $36.2 \pm 1.8$ & \\
\hline$\lambda_{\mathrm{bs}}$ & $170.6 \pm 1.2$ & \\
\hline$\varphi_{\mathrm{bs}}$ & $8.9 \pm 5.9$ & \\
\hline$T_{\mathrm{c}}$ & $5946 \pm 12$ & $5946 \pm 13$ \\
\hline$F_{\mathrm{h}}$ & $1.016 \pm 0.001$ & $1.017 \pm 0.001$ \\
\hline$i\left[{ }^{\circ}\right]$ & $68.9 \pm 0.1$ & $69.9 \pm 0.1$ \\
\hline$\Omega_{\mathrm{h}, \mathrm{c}}$ & $2.263 \pm 0.002$ & $2.261 \pm 0.002$ \\
\hline$\Omega_{\text {in }}, \Omega_{\text {out }}$ & $2.2962,2.1529$ & $2.2962,2.1529$ \\
\hline$f_{\text {over }}[\%]$ & 23.16 & 24.59 \\
\hline$R_{\mathrm{h}, \mathrm{c}}[D=1]$ & $0.486,0.250$ & $0.486,0.250$ \\
\hline$L_{\mathrm{h}} /\left(L_{\mathrm{h}}+L_{\mathrm{c}}\right)$ & $0.798[\mathrm{~B}] ; 0.797[\mathrm{~V}]$ & $0.805[\mathrm{~B}] ; 0.803[\mathrm{~V}]$ \\
\hline
\end{tabular}

Note: $n$ : total number of the $B$ and $V$ observations, $\Sigma(\mathrm{O}-\mathrm{C})^{2}$ : final sum of squares of residuals between observed (LCO) and synthetic (LCC) light-curves, $\sigma_{\mathrm{rms}}$ : root-mean-square of the residuals, $q=m_{\mathrm{c}} / m_{\mathrm{h}}$ : mass ratio of the components, $T_{\mathrm{h}, \mathrm{c}}$ : temperature of the hotter primary and cooler secondary, $\beta_{\mathrm{h}, \mathrm{c}}$ : $A_{\mathrm{h}, \mathrm{c}}, f_{\mathrm{h}, \mathrm{c}}$ : gravity-darkening exponents, albedos and nonsynchronous rotation coefficients of the components respectively, $A_{\mathrm{s}}, \theta_{\mathrm{s}}, \lambda_{\mathrm{s}}$ and $\varphi_{\mathrm{s}}$ : spot temperature coefficient, angular dimension, longitude and latitude (in arc degrees), $F_{\mathrm{h}}$ : filling factor for the critical Roche lobe of the hotter primary, $i\left[{ }^{\circ}\right]$ : orbit inclination (in arc degrees), $L_{3}$ : third light, $\Omega_{\mathrm{h}, \mathrm{c}}, \Omega_{\mathrm{in}}, \Omega_{\mathrm{out}}$ : dimensionless surface potentials of the components and of the inner and outer contact surfaces respectively, $f_{\text {over }}[\%]$ : degree of overcontact, $R_{\mathrm{h}, \mathrm{c}}$ : polar radii of the components in units of the distance between the component centres and $L_{\mathrm{h}} /\left(L_{\mathrm{h}}+L_{\mathrm{c}}+\right.$ $\left.L_{3}\right)$ : luminosity $(B ; V)$ of the less-massive hotter star (including cool spot).

bright spot at longitude $\lambda_{\mathrm{bs}} \sim 171^{\circ}$ produces a slight light curve asymmetry. The noticeable phase dependence of the colour curve $B-V$ (Figure 3 ) is mainly caused by the eclipses of components, but its form is also partly affected by the presence of the bright spot region.

\section{Conclusions}

\subsection{XY Leo}

A summary of our results, given in Table 5, shows that the adopted Roche model with a cool active region on the less-massive component of XY Leo can successfully simulate the observed light curves. Synthetic light 
curves obtained in simultaneous analysis of $B V$ band light curves fit the observations very well. Without this active region we have a fit of a poorer quality, especially in the phase interval $0.0-0.3$.

The solution describes the XY Leo system as a W-type W UMa contact binary with a shallow degree of overcontact $f_{\text {over }} \sim 2.4 \%$ and the orbital inclination $i \sim 68^{\circ}$ responsible for partial eclipses. There is also a relatively large temperature difference between the components $\left(\Delta T=T_{\mathrm{h}}-T_{\mathrm{c}} \sim 330 \mathrm{~K}\right)$. The absolute parameters of the system show that the less-massive hotter component of XY Leo seems to be overluminous and of larger radius with respect to its mass. The bolometric absolute magnitude of the more-massive component is in agreement with that of low mass stars presented by Popper (1980) and Dorman et al. (1989), but the less-massive hotter component is brighter for about 1.6 mag. According to Hrivnak (1985) this is due to the energy transfer from the more-massive to the lessmassive component. Recently, Wang (1994) proposed an explanation for W-type phenomenon based on thermalrelaxation-oscillation (TRO) theory (Lucy 1976; Flannery 1976; Robertson \& Eggleton 1977; Yakut \& Eggleton 2005). According to this, within W-type contact systems the hotter secondary component is in contraction phase to its appropriate ZAMS radius, and its overluminosity is the consequence of the conversion of the gravitational energy into heat during that contraction. This is why we have a weak overcontact configuration in W-type systems. In contrary to W-type, A-type W UMa system (where the secondary component is expanding slowly toward its equilibrium configuration) shows distinctive overcontact configuration with larger fill-out factors.

The cool spot area on the less-massive (hotter) star, at the longitude $\lambda_{\mathrm{s}} \sim 10^{\circ}$, which produces a slight asymmetry of the light curves may be a consequence of a solar-type activity.

By combining our photometric solution with the spectroscopic elements given by Barden (1987) we obtained component masses $\mathcal{M}_{\mathrm{h}}=0.50 \mathcal{M}_{\odot}, \mathcal{M}_{\mathrm{c}}=0.82 \mathcal{M}_{\odot}$ and mean radii $\mathcal{R}_{\mathrm{h}}=0.68 \mathcal{R}_{\odot}, \mathcal{R}_{\mathrm{c}}=0.85 \mathcal{R}_{\odot}$

Our estimated parameters of this system are in a relatively good agreement with the results given in Yakut et al. (2003).

\subsection{EE Cet}

A summary of our results, given in Table 6, shows that the adopted Roche model can successfully simulate the observed light curves. Synthetic light curves obtained by simultaneous analysis of the $B V$ band light curves fit the observations very well.

The solution describes the EE Cet system as a highovercontact configuration $\left(f_{\text {over }} \sim 33 \%\right)$ with orbital inclination $i \sim 79^{\circ}$. We must note that the observed light curves were contaminated by the third light effect (we adopted $L_{3} \sim 0.54$ ), which can make a large impact on the system parameters, particularly the degree of overcontact and orbit inclination estimates.
We obtained the temperature difference $\left(\Delta T=T_{\mathrm{h}}-\right.$ $\left.T_{\mathrm{c}} \sim 220 \mathrm{~K}\right)$, with the secondary star being the hotter one. This is typical for the W-type systems. The slight asymmetry of the light curves can be explained by a cool spot located on the primary star, which probably shows a kind of the solar-type spot activity. However, we have to say that the nature of the asymmetry is not quite clear. Namely, the northern visual companion of ADS 2163, which contributes in total brightness of EE Cet system by $54 \%\left(L_{3} \sim 0.54\right)$ may be a photometric variable. This assumption follows from paper by Rucinski et al. (2002) in which the authors mentioned that this star is a close spectroscopic binary of about the same spectral type. In this case a comparable variability over $0.3-0.4$ days has to be expected. Evidently, in this case the asymmetry of EE Cet light curves could be a consequence of the photometric variability of the neighboring visual companion which contaminates the EE Cet radiation, and thus the 'spot effect' is superfluous. To learn the nature of EE Cet light curves asymmetry we need further CCD photometric observations capable to separate EE Cet from its companion. We expect that these future CCD photometric observations would allow better insight in the characteristics of components and more reliable estimates of EE Cet parameters, and perhaps of the neighboring system as well. This would put even more tight constraints on properties and parameters of these two close binary systems.

By combining our photometric solution with the spectroscopic elements given by Rucinski et al. (2002), we obtained component masses $\mathcal{M}_{c}=1.37 \mathcal{M}_{\odot}$, $\mathcal{M}_{h}=0.43 \mathcal{M}_{\odot}$ and mean radii $\mathcal{R}_{c}=1.35 \mathcal{R}_{\odot}$, $\mathcal{R}_{h}=0.82 \mathcal{R}_{\odot}$

\section{$4.3 A Q P s C$}

The solution, given in Table 7, describes the AQ Psc system as a A-type W UMa contact binary with overcontact degree $f_{\text {over }} \sim 23 \%$ and orbital inclination $i \sim 68.9^{\circ}$, producing partial eclipses.

Although the masses of the components differ considerably $\left(q=m_{\mathrm{c}} / m_{\mathrm{h}}=0.226\right)$, we obtained the relatively small temperature difference between the components $\left(\Delta T=T_{\mathrm{h}}-T_{\mathrm{c}} \sim 150 \mathrm{~K}\right)$. This suggests a significant energy transfer from the hotter (moremassive) primary onto the cooler (less-massive) secondary through the connecting neck of the common envelope. It was shown that the Roche model, that includes bright spot area located near the neck region on the secondary, can successfully simulate the observed light curves. This bright spot (with longitude $\lambda_{\mathrm{bs}} \sim 171^{\circ}$ ), produces a slight asymmetry of the light curves, and can be explained as a consequence of the thermal energy exchange between the components. Without this active region we have a fit of a poorer quality, especially in the phase interval $0.3-0.4$. 
By combining our photometric solution with the spectroscopic elements given by Lu \& Rucinski (1999), we obtained component masses $\mathcal{M}_{\mathrm{h}}=1.65 \mathcal{M}_{\odot}, \mathcal{M}_{\mathrm{c}}=0.37 \mathcal{M}_{\odot}$ and mean radii $\mathcal{R}_{\mathrm{h}}=1.69 \mathcal{R}_{\odot}, \mathcal{R}_{\mathrm{c}}=0.88 \mathcal{R}_{\odot}$

\subsection{Final Remarks}

The light-curve analyses of XY Leo, EE Cet and AQ Psc was made by applying the inverse-problem method of Djurašević (1992b), based on the modified Marquardt (1963) algorithm. The results for the systems XY Leo and AQ Psc are very much in line with previous results reported in the literature and obtained by different methods. In Table 8 we present some important physical parameters of the analysed systems, derived by combining our photometric solutions with spectroscopic elements given by Barden (1987), Rucinski et al. (2002) and Lu \& Rucinski (1999).

All analysed light curves show small asymmetries. The asymmetry is mainly seen as a difference in the level of the two maxima (the O'Connel effect). This asymmetry is usually attributed to spots. By inclusion of spots in the Roche model one can successfully fit the observed light curves. Nevertheless, the question of the uniqueness of the solution remains open in many cases. Analysis of asymmetric light curves thus requires a special attention. A discussion on some of these problems was given by Yakut \& Eggleton (2005).

In some cases, treating albedos as free parameters can help us in choosing an adequate working hypothesis about the nature and location of spots on either of the two components. By testing several different hypotheses we can finally opt for the one that seems most adequate for describing the system, in the light of all available information. Even then, however, other solutions cannot be excluded.

Our analysis indicates the existence of cool spot regions on W-type systems XY Leo and EE Cet, and a bright spot located close to the neck region of A-type system AQ Psc, presumably a consequence of the energy

Table 8. Some physical parameters of the systems, derived by combining our photometric solutions with spectroscopic elements

\begin{tabular}{llll}
\hline Quantity & XY Leo & EE Cet & AQ Psc \\
\hline $\mathcal{M}_{\mathrm{h}}\left[\mathrm{M}_{\odot}\right]$ & $0.50(4)$ & $0.43(2)$ & $1.65(4)$ \\
$\mathcal{M}_{\mathrm{c}}\left[\mathrm{M}_{\odot}\right]$ & $0.82(9)$ & $1.38(4)$ & $0.37(2)$ \\
$\mathcal{R}_{\mathrm{h}}\left[\mathrm{R}_{\odot}\right]$ & $0.68(1)$ & $0.82(1)$ & $1.70(2)$ \\
$\mathcal{R}_{\mathrm{c}}\left[\mathrm{R}_{\odot}\right]$ & $0.85(2)$ & $1.35(2)$ & $0.88(1)$ \\
$T_{\mathrm{h}}[\mathrm{K}]$ & 4850 & $6314(12)$ & 6095 \\
$T_{\mathrm{c}}[\mathrm{K}]$ & $4524(10)$ & 6095 & $5946(12)$ \\
$\log g_{\mathrm{h}}$ & $4.48(12)$ & $4.25(6)$ & $4.20(5)$ \\
$\log g_{\mathrm{c}}$ & $4.49(16)$ & $4.32(5)$ & $4.12(6)$ \\
$M_{\mathrm{bol}}^{\mathrm{h}}$ & $6.40(12)$ & $4.83(6)$ & $3.41(7)$ \\
$M_{\mathrm{bol}}^{\mathrm{c}}$ & $6.20(12)$ & $3.90(8)$ & $4.94(8)$ \\
$a_{\text {orb }}\left[\mathrm{R}_{\odot}\right]$ & $1.99(3)$ & $2.69(2)$ & $3.24(3)$ \\
\hline
\end{tabular}

transfer from the more-massive primary to the lessmassive secondary. According to the TRO theory, the less-massive star expands afterwards which leads to a net transfer of mass from a less-massive secondary back to the primary, the orbit widening, and the loss of contact at the end of the cycle. The secondary starts to contract to its ZAMS radius and its temperature slightly increases (Wang 1994). XY Leo, and perhaps EE Cet, may be in this second stage of TRO cycle. The question is whether this situation applies to all W-type and A-type systems.

Nuclear evolution, mass transfer and mass loss by stellar winds play important roles in the evolution of close binaries (DCBs, NCBs and LTCBs). The evolution of late-type close binary systems is discussed in details by Yakut \& Eggleton (2005). The evolution of the A- and W-type W UMa systems, similar to those studied in this paper is discussed by Gazeas \& Niarchos (2006), Stepien (2006) and Yakut \& Eggleton (2005). Accurate fundamental stellar parameter from both spectroscopic and photometric observations are especially important for gaining more knowledge about $\mathrm{CB}$ systems and their evolution. Providing these parameters for XY Leo, EE Cet and AQ Psc was the main aim of our paper.

\section{Acknowledgments}

This work has been supported by the Ministry of Science and Environmental Protection of Serbia through the project No. 146003 "Stellar and Solar Physics". The authors would like to acknowledge the support of UNESCO-ROSTE for the collaboration among the countries of SE Europe. They would also like to thank the director of the Rozhen NAO for the observing time. This research made use of the SIMBAD database, operated at CDS, Strasbourg, France. The authors are indebted to the referee for her/his careful critiques, constructive suggestions and comments.

\section{References}

Barden, S. C. 1987, ApJ, 317, 333

Claret, A. 2000, A\&A, 363, 1081

Djurašević, G. 1992a, Ap\&SS, 196, 241

Djurašević, G. 1992b, Ap\&SS, 197, 17

Djurašević, G., Zakirov, M., Hojaev, A. \& Arzumanyants, G. 1998, A\&AS, 131, 17

Djurašević, G., Albayrak, B., Tanriverdi, T. \& Erkapić, S. 2004a, A\&A, 415, 283

Djurašević, G., Albayrak, B., Selam, S. O., Erkapić, S. \& Senavci, H. V. 2004b, NewA, 9, 425

Dommanget, J. \& Nys, O. 2002, Observations et Travaux, 54, 2

Dorman, B., Nelson, L. A. \& Chau, W. Y. 1989, ApJ, 342, 1003

ESA, 1997, The Hipparcos and Tycho Catalogs, SP-1200

Flannery, B. P. 1976, ApJ, 205, 217

Gazeas, K. D. \& Niarchos, P. G. 2006, MNRAS, 370, L29

Gehlich, U. K., Prolss, J. \& Wehmeyer, R. 1972, A\&A, 18, 477

Hilditch, R. W. 1981, MNRAS, 196, 305

Høg, E., Fabricius, C., Makarov, V. V., Urban, S., Corbin, T., Wycoff, G., Bastin, U., Schwekendiek, P. \& Wicenec, A. 2000, A\&A, 355, L27

Hoffmeister, C. 1934, AN, 253, 193

Hrivnak, B. J. 1985, ApJ, 290, 696 
Kaluzny, J. \& Pojmanski, G. 1983, AcA, 33, 277

Kwee, K. K. \& van Woerden, H. 1956, BAN, 12, 327

Lampens, P., Oblak, E., Duval, D. \& Chareton, M. 2001, A\&A, 374,132

Lu, W. \& Rucinski, S. M. 1999, AJ, 118, 515

Lucy, L. B. 1967, ZA, 65, 89

Lucy, L. B. 1976, ApJ, 342, 1003

Marquardt, D. W. 1963, J. Soc. Ind. Appl. Math., 11, 431

Pan, L. \& Cao, M. 1998, Ap\&SS, 259, 285

Popper, D. M. 1980, ARA\&A, 18, 115

Pribulla, T. \& Rucinski, S. M. 2006, AJ, 131, 2986

Robertson, J. A. \& Eggleton, P. P. 1977, MNRAS, 179, 359
Rucinski, S. M. 1999, AcA, 19, 245

Rucinski, S. M., Lu, W., Capobianco, C. C., Mochnacki, S. W., Melvin Blake, R. \& Thomson, J. R. 2002, AJ, 124, 1738

Sarma, M. B. K. \& Radhakrishnan, K. R. 1982, IBVS, 2073

Stepien, K. 2006, AcA, 56, 199

Wang, J. M. 1994, ApJ, 434, 277

Wilson, R. E. \& Devinney, E. J. 1971, ApJ, 166, 605

Yakut, K., Ibanoglu, C., Kalomeni, B. \& Degirmenci, O. L. 2003, A\&A, 401, 1095

Yakut, K. \& Eggleton, P. P. 2005, ApJ, 629, 1055

Yamasaki, A. 1981, Ap\&SS, 77, 75

Yamasaki, A. 2005, Ap\&SS, 296, 277 